\title{
A High Bandwidth Modified Regulated Cascode TIA for High Capacitance Photodiodes in VLC
}

\begin{abstract}
This paper proposes, for the first time, a modification of the conventional regulated cascode (RGC) amplifier which improves its tolerance to ultra-high photodiode capacitances. In conventional RGCs, there is a Miller capacitance introduced by the base-collector capacitance of the common base stage, limiting bandwidth gain and potentially result in amplifier instability. Here, we eliminate these undesirable characteristics by introducing an additional cascode stage that nullifies the Miller capacitance. We show that exceptional improvements in bandwidth can be made, which is important in the context of visible light communications systems, where using large-area (large capacitance) photodiodes is desirable.

Index Terms-Optical receiver, regulated cascode, transimpedance amplifier, visible light communication
\end{abstract}

\section{INTRODUCTION}

Transimpedance amplifiers (TIAs) plas a critical role in optical receivers, converting the photodiode current into an amplified voltage. Numerous reports have been published in the literature on the design of TIAs for fibre-optic communication systems at high data rates [1-3]. However, in the vast majority of reports, the designs involve photodiode capacitances $\left(c_{p d}\right)$ of typically just a few picoFarads $(\mathrm{pF})$, which is particularly low in comparison to the values encountered in visible light communication (VLC) systems. Designs have been reported that utilise key bandwidth extension techniques, such as low input impedance amplifiers featuring common base (CB) $[2,4,5]$ or regulated cascode (RGC) $[6,7]$ configurations. Different realisations of the RGC have been reported, with some featuring additional bandwidth optimisation techniques such as inductive [8] and capacitive peaking [9]. These techniques lead to a further extension of the amplifier bandwidth. Others have focused on power and stability optimisation, while maintaining high bandwidths $[10,11]$.

In the context of VLC systems, the basic TIA design principles are still valid, however some idiosyncrasies of such systems introduce additional design constraints. Firstly, the nature of the free space channel mainly relies on line-ofsight and drops with the square of the distance, restricting the optical power at the receiver. Secondly, the vast majority of photodiodes operating in the visible range are silicon, which has higher sensitivity at infrared wavelengths, and relatively poor performance in the visible range, limiting the converted photocurrent. Therefore, in order to boost the optical signal detection in VLC systems, a combination of high gain TIAs with either large photoactive area detectors [12-14] or new materials $[15,16]$ are required. However, large area photodetectors are typically associated with high intrinsic junction capacitance, which imposes a limit on the receiver bandwidth, particularly for high input impedance amplifiers.

Furthermore, with advances in photodetectors using polymer-based materials, devices with responsivity higher than that of silicon in the visible range are emerging [16], giving rise to a new class of photodiodes well suited to VLC systems. The disadvantage of these devices is their significantly lower charge transport compared to silicon (at least three orders of magnitude), and hence, there is a much stricter bandwidth limitation. As a result, there is a clear need for low input impedance TIAs to approach optimal performance using the new materials or large area silicon devices $[15,16]$. For instance, the only reported fully organic optical link using OPDs used a commercial $50 \Omega$ TIA and supported a transmission rate of only $350 \mathrm{~kb} / \mathrm{s}$ without any advanced digital signal processing [15]. A direct method for yielding higher transmission speeds in these links, which are restricted by the photodetector, is to employ a low input impedance TIA.

In this work, we develop a new low input impedance RGCbased TIA that works even with exceptionally high photodiode capacitances in the context of VLC systems that require large area devices. The conventional RGC (CRGC) performance is examined with high $c_{p d}$, while some design parameters such as the transistor transconductance $g_{m 1}$ and $g_{m 2}$ are varied. We develop and propose a new TIA configuration based on the RGC, which we call the modified RGC (MRGC), that significantly improves tolerance to high photodiode capacitances and improves stability. The CRGC is modified by eliminating the Miller capacitance seen at the input as a result of the base collector capacitance $\left(c_{\mu}\right)$ of the $\mathrm{CB}$ stage [17]. The Miller capacitance at the input interplays with the large photodiode capacitance, hence dictating the bandwidth and stability of the amplifier. Hence, we eliminate the Miller capacitance by introducing a cascode configuration, and therefore provide better isolation to large photodiode capacitance in addition to improved stability.

\section{DESIGN OF THE TRANSIMPEDANCE AMPLIFIER}

\section{A. Conventional RGC}

The CRGC is a commonly used configuration in high speed TIAs. Fig. 1(a) shows the CRGC TIA, a modification of the common base amplifier (Q1) that offers higher transconductance by means of a common emitter stage (Q2). The input resistance of the CRGC is lower than the $\mathrm{CB}$ by a factor equal to the voltage gain stage provided by the feedback of the common emitter stage [6]. To achieve wideband operation, the transconductance has to be maximised in order to reduce 
the input impedance. A simplified equivalent circuit is derived for the CRGC in Fig. 1(a) to examine the frequency-dependent behaviour with a large photodiode capacitance as the transconductance is increased. The model takes into account the effect of the two transistor transconductance transconductance $g_{m}$, resistive loads $r_{1}$ and $r_{2}$. Capacitances $c_{i n}=c_{p d}+c_{\pi 2}$, $c_{t}=c_{\pi 1}+c_{\mu 2}$ and $c_{\mu 2}$ as shown in Fig. 2. Nodal analysis is applied to the equivalent circuit to give the input resistance and a second-order transfer function as shown in (1) and (2) respectively.

$$
\begin{aligned}
& Z_{i n}(0) \approx \frac{1}{g_{m 1}\left(1+g_{m 2} r_{2}\right)} \\
& A_{z}=A_{z}(0) \frac{1}{1+\frac{s}{w_{n} Q}+\frac{s^{2}}{w_{n}^{2}}}
\end{aligned}
$$

where $A_{z}(0)$ is the DC transimpedance gain, $w_{n}$ is the undamped natural frequency and $Q$ is the complex pole pair $Q$-factor.

The RGC transfer function is composed of a zero and two real poles; the input and output poles which form a complex pole described by (3) in terms of the damping factor $\zeta=\frac{1}{2 Q}$. Meanwhile, the zero is at much higher frequency than the complex pole, so it can be neglected [18]. Finally, $A_{z}(0)$ and $w_{n}$ are respectively expressed as:

$$
w_{n}=\sqrt{\frac{p_{1,2}=-\zeta \omega_{n} \pm \omega_{n} \sqrt{1-\zeta^{2}}}{A_{z}(0) \approx r_{1}}}
$$

where $A=g_{m 2} r_{2}$ and $Q$ is given by:

$$
Q=\frac{g_{m 1}(1+A)}{w_{n}\left(c_{i n}+c_{t}(1+A)+c_{\mu 1} g_{m 1}\left(r_{1}+r_{2}+A r_{1}\right)\right)}
$$

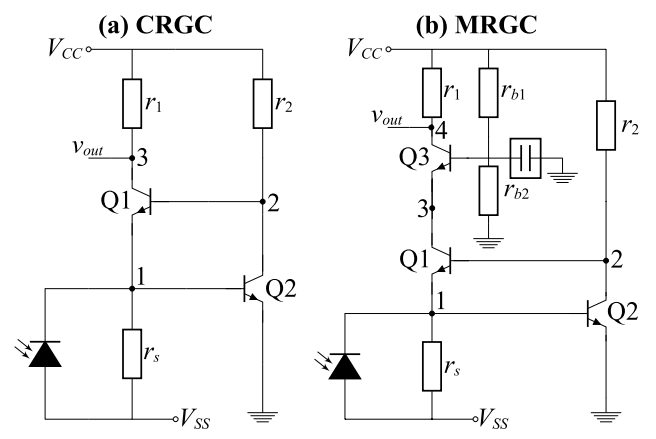

Fig. 1. Circuit diagram for (a) CRGC and (b) MRGC

\section{B. Modified RGC}

In a CRGC, the signal from the collector of Q2 to the base of Q1 sees the transistor Q1 as a common emitter stage. Therefore, the base collector capacitance $\left(c_{\mu 1}\right)$ of Q1 is seen as Miller capacitance at the input of the RGC. In other words, a Miller capacitance value equal to $c_{\mu 1}$ multiplied by the voltage gain of of Q1 is seen at the CRGC input. The voltage gain of Q1 is equal to $g_{m 1} r_{1}$. This Miller capacitance interplays with

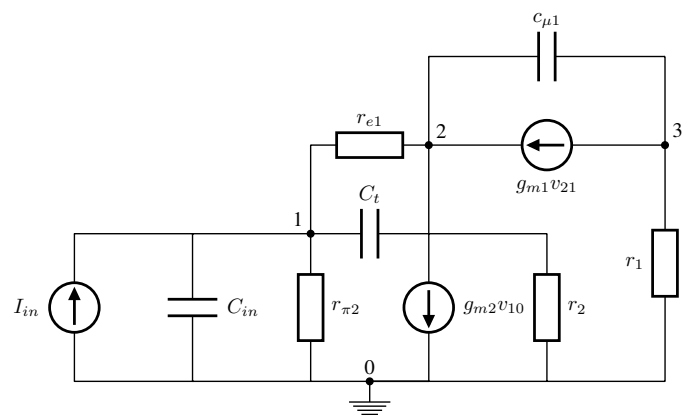

Fig. 2. Simplified small signal model of the CRGC

the large photodiode capacitance, generating a complex pole that dictates the bandwidth and stability of the RGC.

Fig. 1(b) shows a new TIA configuration based on the RGC, i.e. the MRGC. In this circuit the Miller capacitance resulting from the base collector capacitance $\left(c_{\mu 1}\right)$ of $\mathrm{Q} 1$ is eliminated through a cascode configuration that adds a common base stage Q3. Therefore, the signal flowing from the collector of Q2 to the base of Q1 is not limited by the Miller effect of the base collector capacitor $c_{\mu 1}$. In other words, the proposed RGC modification extends the bandwidth of the RGC by neutralising the impact of the Miller capacitance on the frequency of the complex pole, thus shifting the location of the complex pole to a higher frequency. A simplified model of the proposed MRGC has been derived and is illustrated in Fig. 3. The model considers the capacitances $c_{i n}=c_{p d}+c_{\pi 2}$, $c_{t}=c_{\pi 1}+c_{\mu 2}, c_{\mu 1}$ and $c_{\mu 3}$. Similar to the CRGC, the frequency response of the MRGC can be approximated by (1) where the zero is still neglected. The input resistance and $A_{z}(0)$ remain the same as the CRGC as shown in (1) and (2) respectively. Similarly, the complex pole can be evaluated as in (3). On the other hand, $w_{n}$ and $Q$ are respectively expressed as:

$$
\begin{gathered}
w_{n 2}=\sqrt{\frac{g_{m 1}\left(1+A+g_{m 1} r_{\pi 3}+g_{m 2} g_{m 1} r_{2} r_{\pi 3}\right)}{b}} \\
b=\left(c _ { i n } \left(c_{\pi 1} r_{2}+c_{\mu 1} r_{2}+c_{\mu 3} r_{1}+c_{\mu 1} r_{\pi 3}+c_{\pi 1} g_{m 1} r_{2} r_{\pi 3}+\right.\right. \\
\left.c_{\mu 1} g_{m 1} r_{2} r_{\pi 3}+c_{\mu 3} g_{m 1} r_{1} r_{\pi 3}+c_{\mu 1} g_{m 1} r_{2} r_{\pi 3}\right)+c_{\pi 1} c_{\mu 1} x+ \\
\left.c_{\pi 1} c_{\mu 3} r_{1}\left(1+A+A g_{m 1} r_{\pi 3}+g_{m 1} r_{\pi 3}\right)+c_{\mu 1} c_{\mu 3} g_{m 1} r_{1} x\right) \\
Q=\frac{g_{m 1}\left(1+A+g_{m 1} r_{\pi 3}+g_{m 2} g_{m 1} r_{2} r_{\pi 3}\right)}{d w_{n 2}} \\
d=\left(c_{i n}\left(1+g_{m 1} r_{\pi 3}\right)+c_{\pi 1}\left(1+A+c_{\pi 1} g_{m 1} r_{\pi 3}+A g_{m 1} r_{\pi 3}\right)\right. \\
+c_{\mu 1} g_{m 1}\left(r_{2}+r_{\pi 3}+g_{m 1} r_{2} r_{\pi 3}+A r_{\pi 3}\right)+ \\
\left.c_{\mu 3} g_{m 1} r_{1}\left(1+g_{m 1} r_{\pi 3}+A g_{m 1} r_{\pi 3}+A\right)\right)
\end{gathered}
$$

\section{RESULTS}

The performance of the CRGC and the MRGC with a photodiode capacitance set to $C_{p d}=4 \mathrm{nF}$ is analysed in terms of its stability and bandwidth. According to the equations derived in Section II increasing $g_{m}$, reduces the input resistance which leads to increased bandwidth. This is 


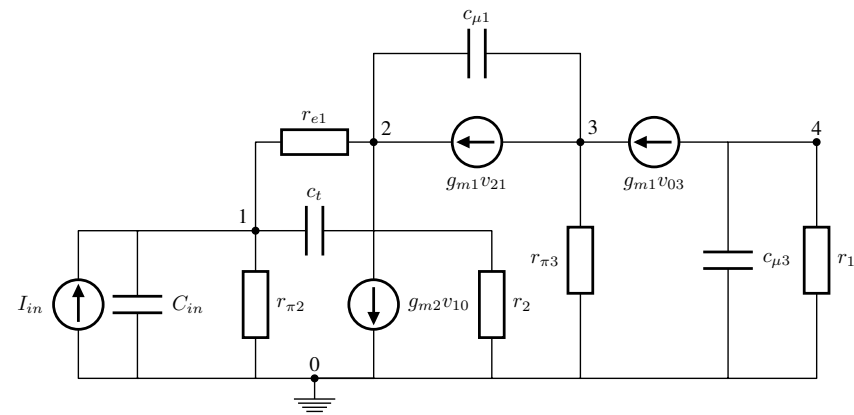

Fig. 3. Small signal model of the MRGC

further highlighted by $w_{n}$ and the complex pole equations, where the value of $w_{n}$ increases with increasing $g_{m}$. This in turn shifts $p_{1}$ to higher frequencies, enhancing both the CRGC and MRGC bandwidths. However, increasing $g_{m}$ has the downside of increasing $Q$, which lowers the phase margin and hence trades the stability of the CRGC amplifier. Therefore, increasing $g_{m}$ enhances the amplifier bandwidth, increases $Q$ and leads to peaking in the frequency response and reduction in the phase margin which could eventually lead to amplifier oscillation. Hence, increasing $g_{m}$ imposes a trade-off between the amplifier stability and bandwidth for the CRGC. On the other hand, increasing $g_{m}$ for the MRGC causes an increase in $w_{n}$ and a comparatively less severe increase of $Q$, while the phase margin reduces less significantly, improving stability with respect to the CRGC, as will be illustrated later.

In order to illustrate the above analysis, the stabilities of the CRGC and the MRGC are examined by plotting the amplifier pole action on the $s$-plane while sequentially varying $g_{m 1}$ and $g_{m 2}$. First, we set $g_{m 2}$ constantly at $40 \mathrm{mS}$ to ensure there is no effect on the phase margin or peaking, while the value of $g_{m 1}$ is varied in the range of $40 \mathrm{mS}$ to $280 \mathrm{mS}$ in steps of $40 \mathrm{mS}$. Next, we constantly set $g_{m 1}=40 \mathrm{mS}$ and vary $g_{m 2}$.

Starting with point (1a) for the case of the CRGC, the case where $g_{m 2}=40 \mathrm{mS}$ and $g_{m 2}$ is varied, Fig. 4 illustrates that increasing the value of $g_{m 1}$ pushes the complex pole towards the unstable region of the $s$-plane as indicated by the arrow. This is because increasing $g_{m 1}$ increases the CB voltage gain, which in turn increases the value of the Miller capacitance. The direct result of which is a reduction in the stability of the amplifier. Meanwhile, in the case of the MRGC with the same constraints of $g_{m 1}$ and $g_{m 2}$, i.e. point (1b), the effect of the Miller capacitance is neutralised, as increasing $g_{m 1}$ pushes the complex pole away from the unstable region. Moreover, the increase in the amplifier bandwidth is demonstrated via the increase in the distance between each of the complex poles from the real axis, which is equivalent to the value of the damped natural frequency $w_{d}$. Therefore, it is clear that in the case of the CRGC (1a), increasing $g_{m 1}$ results in a marginal increase in $w_{d}$, leading to a comparatively minor bandwidth enhancement. On the other hand, the increase in $w_{d}$ in the case of the MRGC (1b) as $g_{m 1}$ increases is significant, resulting in a vastly improved bandwidth which will be illustrated later.
Broadly speaking, it can be inferred from Fig. 4 that increasing $g_{m 1}$ has the opposite effect on the CRGC in comparison to the MRGC.

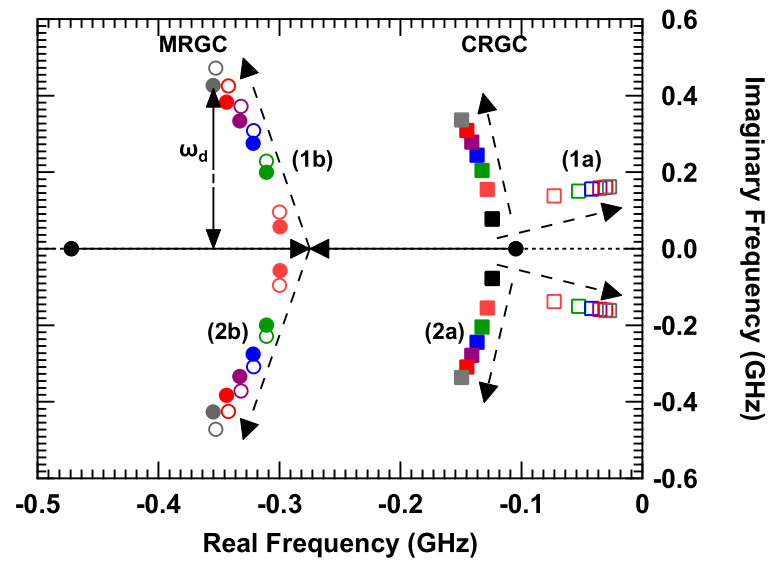

\begin{tabular}{|c|c|c|c|c|c|c|}
\hline & \multicolumn{3}{|c|}{$g_{\mathrm{m} 2}=40 \mathrm{~ms}$} & \multicolumn{3}{|c|}{$g_{m 1}=40 \mathrm{mS}$} \\
\hline & CRGC & & MRGC & & CRGC & MRCG \\
\hline$\square$ & $g_{m 1}=40$ & 0 & $g_{m 1}=40$ & 口 & $g_{m 2}=40$ & $\mathrm{~g}_{\mathrm{m} 2}=40$ \\
\hline$\square$ & $g_{m 1}=80$ & 0 & $g_{m 1}=80$ & 口 & $g_{m 2}=80$ & $g_{m 2}=80$ \\
\hline$\square$ & $g_{m 1}=120$ & 0 & $g_{m 1}=120$ & - & $g_{m 2}=120$ & $g_{m 2}=120$ \\
\hline$\square$ & $g_{m 1}=160$ & 0 & $g_{m 1}=160$ & & $g_{m 2}=160$ & $g_{m 2}=160$ \\
\hline$\square$ & $g_{m 1}=200$ & 0 & $g_{m 1}=200$ & 口 & $g_{m 2}=200$ & $\mathrm{~g}_{\mathrm{m} 2}=\mathbf{2 0 0}$ \\
\hline$\square$ & $g_{m 1}=240$ & 0 & $g_{m 1}=240$ & - & $g_{m 2}=240$ & $g_{m 2}=240$ \\
\hline$\square$ & $g_{m 1}=280$ & 0 & $g_{m 1}=280$ & - & $g_{m 2}=280$ & $g_{m 2}=280$ \\
\hline
\end{tabular}

Fig. 4. Pole action of the CRGC versus the MRGC for different $g_{m 1}$ and $g_{m 2}$ values and $c_{i n}=4 \mathrm{nF}$

On the other hand, seeing $g_{m 1}=40 \mathrm{mS}$ constantly, while increasing $g_{m 2}$ has a similar effect in the case of the CRGC at point (2a) and the MRGC (2b), where the complex pole is pushed away from the unstable region of the $s$-plane in both cases. Nevertheless, it is clear that the complex pole loci for the MRGC sits further away in comparison to the CRGC in terms of the real frequency, due to the elimination of Miller capacitance effect. Recalling that $g_{m 2}$ is the varied while $g_{m 1}$ is set constant at $40 \mathrm{mS}$, the Miller effect observed in the CRGC is not eliminated, but kept constant at $C_{m}=g_{m 1} r_{1} c_{\mu 1}$. Moreover, with regards to bandwidth extension, increasing $g_{m 2}$ in the case of the CRGC (2a) increases $w_{d}$ and therefore increases the amplifier bandwidth more significantly than the previous case. Nevertheless, the bandwidth enhancement is still limited by the Miller capacitance. Meanwhile, in the MRGC case at point (2b) it is observed that increasing $g_{m 2}$ provides a significant bandwidth enhancement. This conclusion is highlighted in Fig. 5 which shows the phase margin for the CRGC and MRGC. The red lines indicate the case where $g_{m 2}$ is varied and $g_{m 1}$ is $40 \mathrm{mS}$, and the blue is the alternative case. From Fig. 5, it is clearly evident that the CRGC amplifier phase margin reduces substantially as the value of $g_{m 1}$ is increased to approximately 20 degrees when $g_{m 1}=280 \mathrm{mS}$. This large reduction is due to an increase in the Miller capacitance seen at the input of the CRGC caused by the increase in the CB Q1 voltage gain. On the other hand, in the MRGC case, the amplifier maintains a reasonably constant phase margin as the value of $g_{m 1}$ is increased, because the effect of the Miller capacitance is eliminated, thus confirming the advantage the additional cascode stage. 


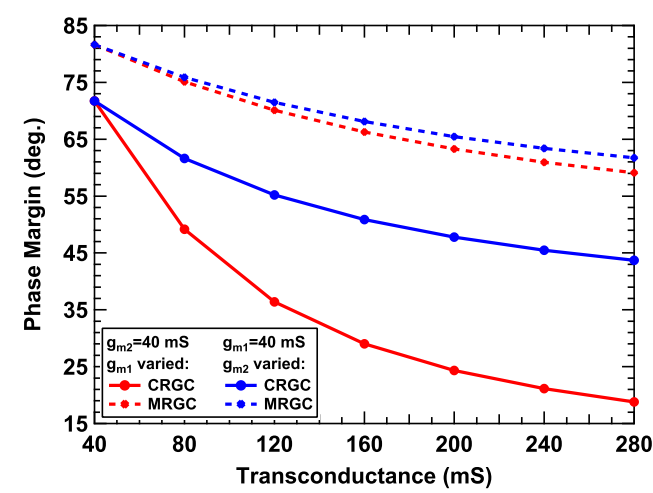

Fig. 5. Phase margin of the CRGC versus the MRGC as the transconductance is increased

Hence, based on the pole action and the phase margin of the two amplifiers it can be concluded that in the case of the CRGC that the marginal gain in the amplifier bandwidth by increasing $g_{m 1}$ is nullified by the increase in the value of the Miller capacitance which compromises the amplifier stability. Therefore, it is best to opt for low values of $g_{m 1}$ to ensure the CRGC is stable. Meanwhile, in the case of MRGC, there is a clear benefit in the elimination of the Miller capacitance in both the amplifier stability and bandwidth. Therefore, the value of $g_{m 1}$ can be increased to achieve higher bandwidth.

In order to capitalise on the conclusions drawn from the complex pole action and the phase margin of the two amplifiers, the transimpedance gain, voltage gain and input impedance of both configurations are shown in Figs. 6(a)-(c). We introduce two new cases; $(i)$ case 1 , where $g_{m 1}=40 \mathrm{mS}$ for the two amplifiers to reduce the effect of the Miller capacitance, ensuring sufficient phase margin and moderate peaking in the case of the CRGC. The value of $g_{m 2}$ is set to $240 \mathrm{mS}$ to achieve the best possible bandwidth, while maintaining a phase margin $>45$ degrees; next (ii) in case 2 , the value of $g_{m 1}=120 \mathrm{mS}$ and $g_{m 2}=240 \mathrm{mS}$. Fig. 6(a) shows that in case 1 the CRGC gives a bandwidth of $71 \mathrm{MHz}$ with a moderate peak, while the MRGC provides a greater bandwidth $(81 \mathrm{MHz})$, yet the response is clearly over-damped which implies that the value of $g_{m 1}$ can be increased for further bandwidth extension. In case 2 , increasing the value of $g_{m 1}$ results in a very sharp peak in the CRGC frequency response and a slight bandwidth extension (of $17 \mathrm{MHz}$ ) over the previous case. On the other hand, for the MRGC, the new values lead to a substantial bandwidth extension of $115 \mathrm{MHz}$, or a total of $196 \mathrm{MHz}$. Therefore, these results confirm that the proposed MRGC configuration provides an additional degree of freedom for the designer, enabling maximisation of $g_{m 1}$, which enables a substantial increase in the bandwidth, with acceptable frequency peak and phase margins.

These results are strongly supported by Fig. 6(b), which shows the voltage gain of each configuration for both cases. The neutralisation of the Miller capacitance is clear for both cases, due to the lower frequency dependence of the voltage gain in both cases. Finally, Fig. 6(c) shows the input
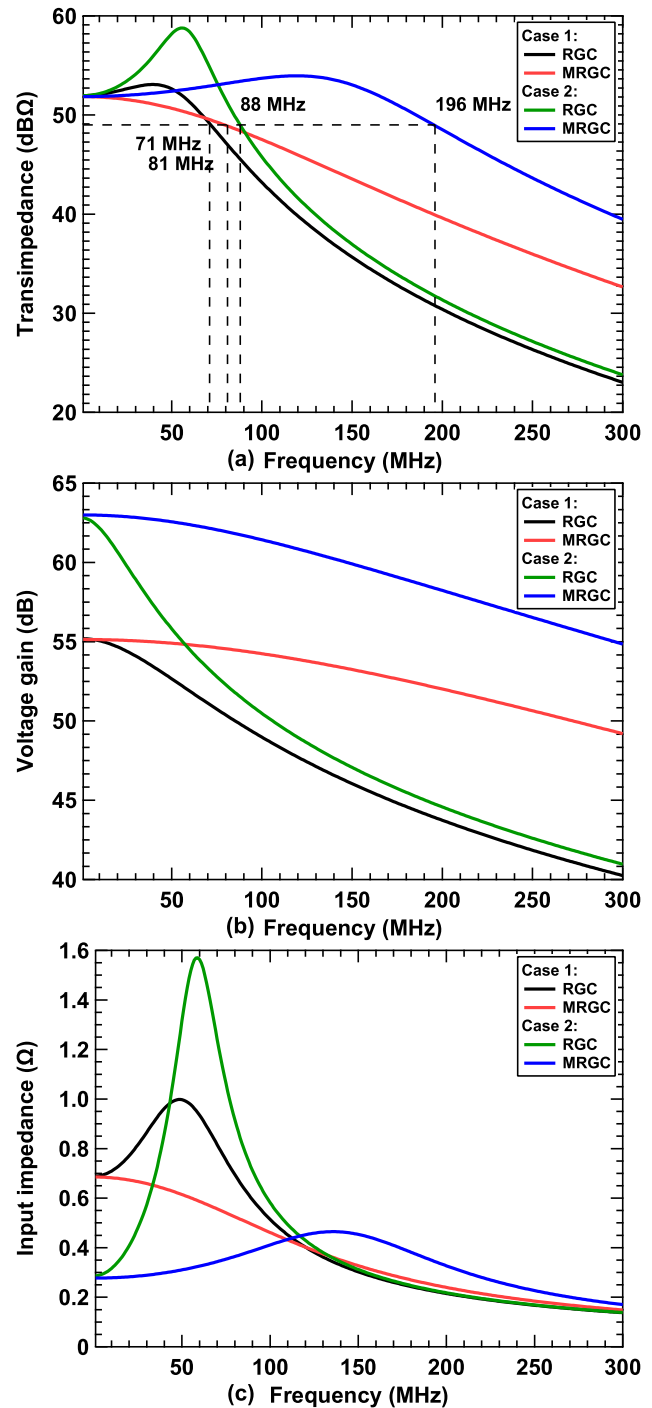

Fig. 6. (a) Transimpedance gain (b) voltage gain and (c) input impedance of the CRGC and the MRGC for two different $g_{m}$ cases

impedance for both configurations, where they are initially equivalent in both cases. Clearly, with increasing frequency, the peaks are suppressed in the MGCR case comparing to the CRGC, which is advantageous. Together, these results show that large area or high capacitance photodiodes can be tolerated in VLC systems, thus enabling both higher signal powers and increased (signal-to-noise ratios), and higher bandwidths.

\section{CONCLUSION}

A modification of the CRGC has been proposed to eliminate the undesirable Miller capacitance effect that results from the base-collector capacitance of the $\mathrm{CB}$ stage. We show that by adding an additional cascode stage, improved tolerance to ultra high photodiode capacitance can be demonstrated. Furthermore, a simplified equivalent circuit has been derived for each of the RGC variations used, to conduct further stability analysis. We show that the MRGC is not only more stable, but it also provides higher bandwidth and flatter voltage and input impedance responses. 


\section{REFERENCES}

[1] B. Moeneclaey, J. Verbrugghe, J. Lambrecht, E. Mentovich, P. Bakopoulos, J. Bauwelinck, and X. Yin, "Design and experimental verification of a transimpedance amplifier for 64-gb/s pam-4 optical links," Journal of Lightwave Technology, vol. 36, no. 2, pp. 195-203, Jan 2018.

[2] S. Jung, J. D. Cressler, J. A. Babcock, G. Cestra, and A. Buchholz, "A design methodology to achieve low input impedance and non-constant gain-bandwidth product in tias for optical communication," in 2013 IEEE International Symposium on Circuits and Systems (ISCAS2013), May 2013, pp. 598-601.

[3] C. Li and S. Palermo, "A low-power, 26-GHz transformer-based regulated cascode transimpedance amplifier in 0.25um SiGe BiCMOS," in 2011 IEEE Bipolar/BiCMOS Circuits and Technology Meeting, Oct 2011, pp. 83-86.

[4] B. Wilson and I. Darwazeh, "Low input resistance transimpedance optical preamplifier for fibre optic local area networks," in IEEE International Symposium on Circuits and Systems (ISCAS), vol. 1988. IEEE, Conference Proceedings, pp. 2531-2534.

[5] S. Giannakopoulos, Z. S. He, I. Darwazeh, and H. Zirath, "Differential common base TIA with $56 \mathrm{~dB}$ Ohm gain and $45 \mathrm{GHz}$ bandwidth in 130 nm SiGe," in 2017 IEEE Asia Pacific Microwave Conference (APMC), vol. 2017. IEEE, Conference Proceedings, pp. 1107-1110.

[6] S. M. Park and C. Toumazou, "A packaged low-noise high-speed regulated cascode transimpedance amplifier using a 0.6 um N-well CMOS technology," in Proceedings of the 26th European Solid-State Circuits Conference, vol. 2000, Conference Proceedings, pp. 431-434.

[7] S. M. Park and H.-J. Yoo, "1.25-Gb/s regulated cascode CMOS transimpedance amplifier for gigabit ethernet applications," IEEE Journal of Solid-State Circuits, vol. 39, no. 1, pp. 112-121, 2004.

[8] M. N. Ahmed, J. Chong, and D. S. Ha, "A $100 \mathrm{gb} / \mathrm{s}$ transimpedance amplifier in $65 \mathrm{~nm}$ cmos technology for optical communications," in 2014 IEEE International Symposium on Circuits and Systems (ISCAS), June 2014, pp. 1885-1888.

[9] Y.-J. F.-T-Chien, "Bandwidth enhancement of transimpedance amplifier by a capacitive peaking design," IEEE Journal of Solid State Circuits, pp. $37-46,1999$.

[10] C. Kromer, G. Sialm, T. Morf, M. L. Schmatz, F. Ellinger, D. Erni, and H. Jackel, "A low-power 20-GHz 52-dB/spl Omega/ transimpedance amplifier in 80-nm CMOS," IEEE Journal of Solid-State Circuits, vol. 39, no. 6, pp. 885-894, June 2004.

[11] B. Abdollahi, B. Mesgari, S. Saeedi, and A. Nabavi, "Stability analysis and compensation technique for low-voltage regulated cascode transimpedance amplifier," Microelectronics Journal, vol. 71, pp. 37 - 46, 2018.

[12] R. L. Aguiar, A. Tavares, J. L. Cura, E. D. Vaaconcelos, L. N. Alves, R. Valadas, and D. M. Santos, "Considerations on the design of transceivers for wireless optical lans," in IEE Colloquium on Optical Wireless Communications (Ref. No. 1999/128), June 1999, pp. 2/1-231.

[13] Z. Ghassemlooy, W. Popoola, and S. Rajbhandari, Optical Wireless Communications. CRC Press, 2013.

[14] J. L. Cura and L. N. Alves, "Bandwidth improvements in transimpedance amplifiers for visible-light receiver front-ends," in 2013 IEEE 20th International Conference on Electronics, Circuits, and Systems (ICECS), Dec 2013, pp. 831-834.

[15] P. A. Haigh, Z. Ghassemlooy, I. Papakonstantinou, F. Arca, S. F. Tedde, O. Hayden, and E. Leitgeb, "A 1-mb/s visible light communications link with low bandwidth organic components," IEEE Photonics Technology Letters, vol. 26, no. 13, pp. 1295-1298, July 2014.

[16] S. F. Tedde, J. Kern, T. Sterzl, J. Fürst, P. Lugli, and O. Hayden, "Fully spray coated organic photodiodes," Nano Letters, vol. 9, no. 3, pp. 980983, 2009, pMID: 19239203.

[17] S. Bashiri, C. Plett, J. Aguirre, and P. Schvan, "A $40 \mathrm{~Gb} / \mathrm{s}$ transimpedance amplifier in $65 \mathrm{~nm}$ CMOS," in IEEE International Symposium on Circuits and Systems (ISCAS), vol. 2010. IEEE, Conference Proceedings, pp. 757-760.

[18] L. B. Oliveira, C. M. Leitao, and M. M. Silva, "Noise performance of a regulated cascode transimpedance amplifier for radiation detectors," IEEE Transactions on Circuits and Systems I: Regular Papers, vol. 59, no. 9, pp. $37-46,2012$. 